\title{
Efficient compound OPO cavity with narrow bandwidth
}

Sheng Wu, Geoffrey A. Blake, Sunny Sun, Jiwu Ling

Sheng Wu, Geoffrey A. Blake, Sunny Sun, Jiwu Ling, "Efficient compound OPO cavity with narrow bandwidth," Proc. SPIE 3263, Nonlinear Optical Engineering, (19 May 1998); doi: 10.1117/12.308348

SPIE Event: Optoelectronics and High-Power Lasers and Applications, 1998, San Jose, CA, United States 


\title{
An efficient compound OPO cavity with narrow bandwidth
}

\author{
Sheng Wu', Geoffrey A. Blake ${ }^{2}$ \\ Sunny Sun, Jiwu Ling ${ }^{3}$ \\ ${ }^{1}$ Division of Chemistry, California Institute of Technology, CA 91125 \\ ${ }^{2}$ Division of Geological and Planetary Science, California Institute of Technology, CA \\ 91125 \\ ${ }^{3}$ CASIX Inc., 1933 South Myrtle Avenue, Monrovia, CA 91016-4854
}

\begin{abstract}
By integrating a free running cavity and a narrow bandwidth cavity, it is possible to create a compound OPO design which possesses the most desirable properties of each. In this example, we have combined a $355 \mathrm{~nm}$ pumped idler-resonated free running type I BBO OPO cavity with a grating-narrowed signal-reinjection arm using only standard, low-cost optics. The output from such an implementation retains the high efficiency and low divergence of the free-running OPO, but its bandwidth is dictated by that of the high resolution cavity.
\end{abstract}

Key words: OPO, compound cavity

OPO cavities which produce high spectral resolution in a compact, rugged design are finding increasing use in a variety of spectroscopic and remote sensing applications ${ }^{1}$. In many commercial approaches, high energy pulses with narrow bandwidths are produced by oscillator/amplifier layouts in which a first, low efficiency, cavity produces narrow band pulses that are subsequently amplified in additional stages ${ }^{2}$ Recently, however, it has become clear that it is possible to combine the oscillation and amplification stages into a single design through the use of compound cavities in which the control of the narrow bandwidth and amplification processes are performed on separate branches of the parametric process ${ }^{3}$.

For example, the compound OPO cavity presented in Figure 1 is the combination of a free running OPO cavity (defined by mirrors Mo, Mi, Ms and Mr) and a narrow bandwidth OPO cavity (formed by the optical elements Mo, Mi, Ms, Grating and Mt). The free running OPO cavity is a singly resonant type I BBO OPO resonated on its idler wave ${ }^{4}$. The narrow bandwidth OPO cavity is also a singly resonant OPO, but it is resonated on the signal wave and builds upon the design of Bosenberg, Pelouch, and Tang (1989) ${ }^{5}$. The properties of the various optical elements are as follows: Mo -- A $0^{\circ}$ high reflector for the pump, in our case the 355nm 3rd harmonic of a Nd:YAG laser; Mi -- A $45^{\circ} 355 \mathrm{~nm}$ pump input coupler; Mr \& Mt -Aluminized metallic mirrors; Ms -- A high reflectivity optic for the signal beam, with high transmission for the idler wave; Grating -- 2700 groves $/ \mathrm{mm}$ grating in a $88^{\circ}$ Littman configuration.

SPIE Vol. $3263 \bullet 0277-786 \times / 98 / \$ 10.00$ 
The pump laser for the compound OPO cavity is the third harmonic (355 nm) of an injection-seeded $\mathrm{Nd}$ :YAG laser, whose pulse width is approximately $10 \mathrm{~ns}$. The BBO crystal is cut for type I phase matching, is $15 \mathrm{~mm}$ in length, and has a broadband AR coating which covers the near-UV and optical range. Under a wide range of conditions, the bandwidth of the compound OPO is dictated by the grating-narrowed arm of the cavity, while the beam quality and the efficiency of the compound OPO cavity is mainly decided by the free running, idler resonated arm -- which has much higher efficiency and therefore dominates the amplification process. By resonating on the idler wave of the free running OPO, a small divergence for the OPO output is obtained. Thus, the compound cavity in Figure 1 is found to possess the same high conversion efficiency and good beam quality of a free running OPO, yet the same bandwidth as a gratingnarrowed OPO.

Table 1 summarizes the efficiency and bandwidth of the compound cavity over the tuning range measured to date. A single grating was used in these experiments, and, including the idler-wave, complete coverage can be obtained from $440-1830 \mathrm{~nm}$. Figure 2 shows the $\sim 0.35 \mathrm{~cm}^{-1}$ bandwidth of the compound cavity at $466 \mathrm{~nm}$ as measured by a Burleigh pulsed spectrum analyzer. Similar measurements across the complete tuning range demonstrate that the compound cavity consistently produces a bandwidth of less than $0.5 \mathrm{~cm}^{-1}$, while the total (signal + idler) efficiency is higher than $40 \%$ when the pump intensity is twice that of the threshold intensity (which is some $25-35 \mathrm{MW} / \mathrm{cm}^{2}$, or about $65-70 \mathrm{~mJ}$ for the $4.5 \mathrm{~mm}$ diameter $355 \mathrm{~nm}$ pump beam).

To summarize, the compound cavity presented herein fully utilizes the signal and idler beams generated in the OPO process to optimize and simplify the OPO design. The results demonstrated to date prove that compound OPO cavities allow efficient generation of widely tunable, narrow bandwidth parametric radiation while easing the demands place on the pump laser.

\section{REFERENCES:}

1. P.I. Ionov, I. Bezel, S.I. Ionov, C. Wittig, Chem. Phys. Lett., 272, 257-264 (1997)

2. B.C. Johnson, V.J. Newell, J.B. Clark, E.S. McPhee, J. Opt. Soc. Am. B, 12, $21222-21227$ (1995)

3. Y. Zhou, Z.Y. Xu, D.Q. Deng, Y.F. Kong, X.A. Zhu, Z.H. Yan, J. Opt. Soc. Am. B, 14, 1496-1500 ( 1997)

4. S. Wu, G.A. Blake, Z.Y. Sun, J.W. Ling, Appl. Optics 36, 5898-5901, (1997)

5. W. R. Bosenberg, W.S. Pelouch and C.L. Tang, Appl. Phys. Lett. 55, 1952-1954 (1989) 
Table 1. Performance of the compound cavity OPO

\begin{tabular}{|l|cccccc|}
\hline Wavelength & $445 \mathrm{~nm}$ & $466 \mathrm{~nm}$ & $540 \mathrm{~nm}$ & $610 \mathrm{~nm}$ & $670 \mathrm{~nm}$ \\
\hline Bandwidth $\left(\mathrm{cm}^{-1}\right)$ & $0.45 \mathrm{~cm}^{-1}$ & $0.35 \mathrm{~cm}^{-1}$ & $0.35 \mathrm{~cm}^{-1}$ & $0.35 \mathrm{~cm}^{-1}$ & $0.30 \mathrm{~cm}^{-1}$ \\
\hline Efficiency & $40^{\circ} \%$ & $42^{\circ} \%$ & $45^{\circ} \mathrm{o}$ & $46^{\circ} \mathrm{o}$ & $44^{\circ} \mathrm{o}$ \\
\hline
\end{tabular}

The pump laser has a beam size of $4.5 \mathrm{~mm}$ in diameter, and when pumped at $135 \mathrm{~mJ}$ ( -2 times above operation threshold). we measured the efficiency

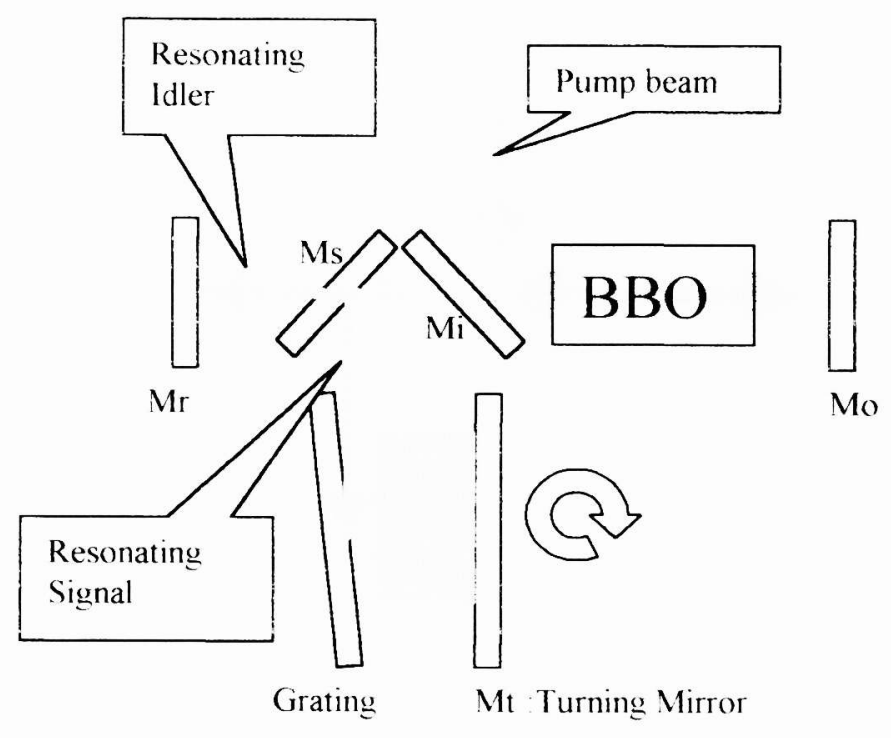

Figure 1. 


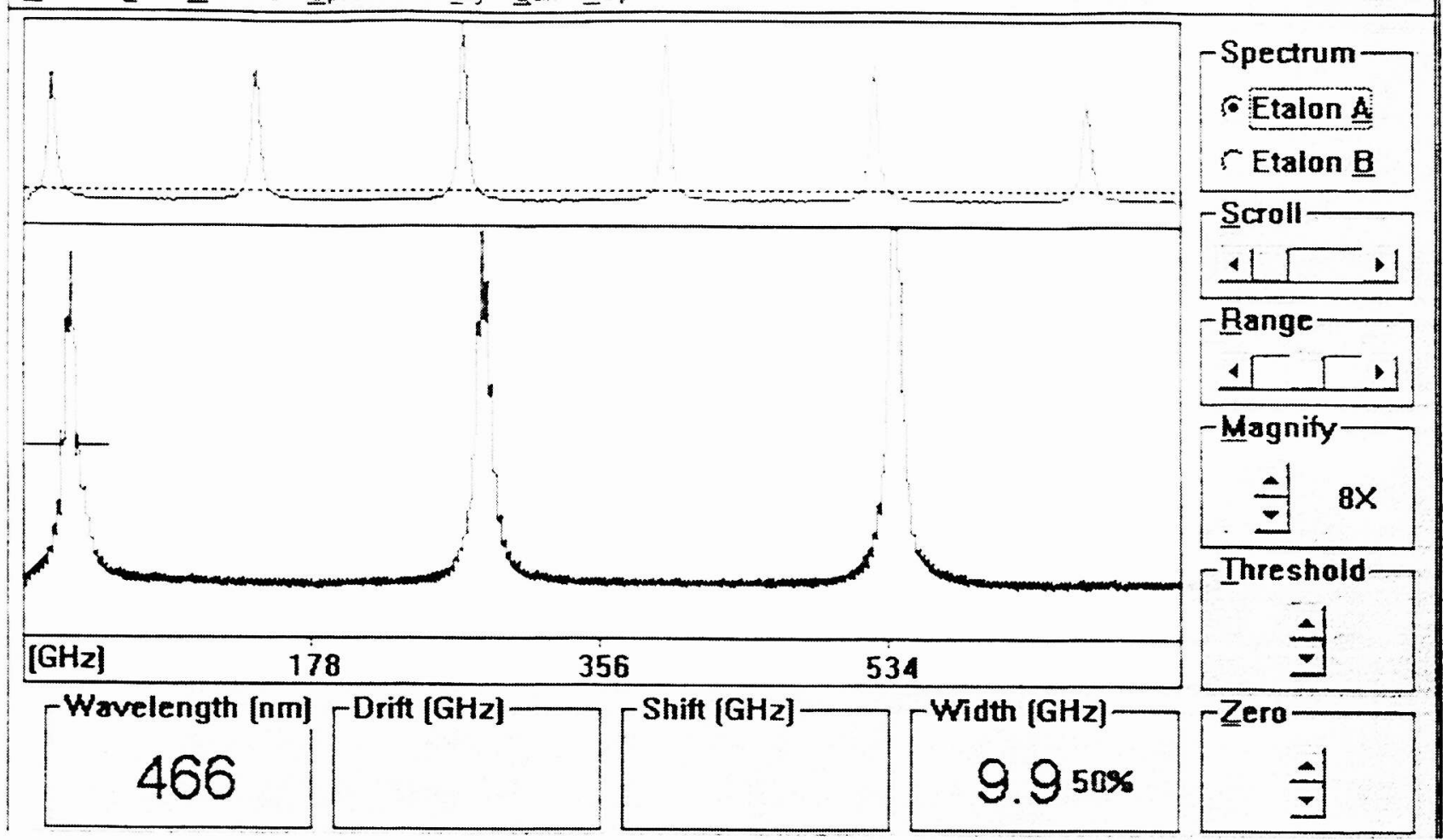

Figure 2. Bandwidth (Full width at half maximum) of the compound OPO cavity, measured with a Burleigh Pulsed Spectrum Analyzer. This is plot is the average result of 50 shots in 5 seconds. 